\title{
Video Article \\ Two Different Real-Time Place Preference Paradigms Using Optogenetics within the Ventral Tegmental Area of the Mouse
}

\author{
Zisis Bimpisidis ${ }^{1}$, Niclas König ${ }^{1}$, Åsa Wallén-Mackenzie ${ }^{1}$ \\ ${ }^{1}$ Department of Organismal Biology, Unit of Comparative Physiology, Uppsala University \\ Correspondence to: Zisis Bimpisidis at zisis.bimpisidis@ebc.uu.se, Åsa Wallén-Mackenzie at asa.mackenzie@ebc.uu.se \\ URL: https://www.jove.com/video/60867 \\ DOI: doi:10.3791/60867
}

Keywords: Behavior, Issue 156, aversion, behavior, conditioning, Cre-Lox, dopamine transporter (DAT), optogenetics, place preference, reward, transgenic mice, vesicular glutamate transporter (VGLUT2)

Date Published: 2/12/2020

Citation: Bimpisidis, Z., König, N., Wallén-Mackenzie, A. Two Different Real-Time Place Preference Paradigms Using Optogenetics within the Ventral Tegmental Area of the Mouse. J. Vis. Exp. (156), e60867, doi:10.3791/60867 (2020).

\section{Abstract}

\begin{abstract}
Understanding how neuronal activation leads to specific behavioral output is fundamental for modern neuroscience. Combining optogenetics in rodents with behavioral testing in validated paradigms allows the measurement of behavioral consequences upon stimulation of distinct neurons in real-time with high spatial and temporal selectivity, and thus the establishment of causal relationships between neuronal activation and behavior. Here, we describe a step-by-step protocol for a real-time place preference (RT-PP) paradigm, a modified version of the classical conditioned place preference (CPP) test. The RT-PP is performed in a three-compartment apparatus and can be utilized to answer if optogenetic stimulation of a specific neuronal population is rewarding or aversive. We also describe an alternative version of the RT-PP protocol, the socalled neutral compartment preference (NCP) protocol, which can be used to confirm aversion. The two approaches are based on extensions of classical methodology originating from behavioral pharmacology and recent implementation of optogenetics within the neuroscience field. Apart from measuring place preference in real time, these setups can also give information regarding conditioned behavior. We provide easyto-follow step-by-step protocols alongside examples of our own data and discuss important aspects to consider when applying these types of experiments.
\end{abstract}

\section{Video Link}

The video component of this article can be found at https://www.jove.com/video/60867/

\section{Introduction}

The implementation of optogenetics, a modern neuroscience experimental tool in which light is used to control neuronal activity, has in recent years led to major advancements in understanding how specific neuronal populations impact behavior ${ }^{1,2,3}$. The outstanding spatial and temporal selectivity of optogenetics allows the establishment of causal relationships between excitation or inhibition of cell groups of interest and behavioral output ${ }^{2,3}$. Spatial selectivity in optogenetics is commonly ensured through the Cre-Lox system in which the activity of Cre recombinase leads to recombination of any DNA sequences present between Lox sites, so called floxed alleles (flanked by lox sites) ${ }^{4}$. The goal with using the Cre-Lox system in optogenetics is to achieve expression of alleles encoding optogenetic opsins in specific neurons of interest while leaving surrounding neurons devoid of expression. Opsins are light-sensitive proteins that upon light-stimulation of specific wave-length allow ion flow that affects neural excitability or influence cellular functions by modulating downstream effector pathways. Novel variants of opsins that differ in action (excitatory, inhibitory, modulatory), mechanism, activation by light wavelength and kinetics properties ${ }^{5}$ are continuously being developed to meet the needs of specific experimental approaches. Regarding excitability, using a depolarizing or hyperpolarizing opsin dictates the activity of the neurons (excitation or inhibition, respectively) upon light-stimulation at a specific wavelength delivered into the brain ${ }^{3}$.

Selective promoter activity directs the expression of Cre recombinase to the neurons of interest. By implementing a floxed allele of the opsin of interest, Cre-mediated recombination will ensure that the opsin is selectively expressed in neurons that co-express Cre recombinase ${ }^{3,6}$. This use of double transgenics to direct spatial selectivity has proven very efficient in optogenetics. Thus, while light-stimulation to activate the opsins is broadly delivered through an intracerebrally implanted optic fiber connected to a light source (LED or laser) ${ }^{3}$, only neurons expressing both Cre recombinase and the floxed opsin allele will respond to this stimulation. The Cre-Lox system in rodents can be achieved in different ways by using only transgenics (both Cre recombinase and the floxed opsin construct are encoded in transgenic animals), only viral injections (DNA constructs for Cre recombinase and the floxed opsin are both delivered via a viral carrier), or a combination of the two (for example, Cre recombinase is encoded by a transgenic animal which is injected with a virus carrying the floxed opsin construct) ${ }^{5}$. The floxed opsin DNA construct is usually cloned in frame with a reporter gene to enable visualization of Cre-mediated recombination in tissue sections. While optogenetics can also be performed in rats, the presented protocols have been generated for mice. For simplicity, mice carrying both Cre recombinase and the floxed opsin will be referred to as "optogenetics mice". In the protocols described below, optogenetics mice have been generated by a mixed transgenic (Cre recombinase under control of two different promoters) and viral (using an adeno-associated virus, AAV, to deliver the floxed opsin DNA construct - in our case ChR2/H134R) approach. Obtaining and maintaining transgenic mouse lines is an essential 
part of the methodology. Cre-driver and floxed opsin transgenic mice can be produced for each purpose, or purchased if commercially available, as can a range of viruses carrying DNA sequences encoding Cre recombinase and floxed opsins in different forms.

Optogenetics coupled with behavioral testing has proven to be a valuable tool to study the role of distinct brain regions, or discrete neuronal populations, in particular types of behavior. In the context of reward-related behavior, optogenetics has enabled the verification of previous findings in the fields of behavioral pharmacology and experimental psychology, and also allowed a new level of spatio-temporally relevant dissection into how certain neurons affect behavior. One method which has been used in several studies to assess reward-related behavior is a modified version of the classical method known as Conditioned Place Preference (CPP). Classical CPP has been used to assess the rewarding or aversive properties of drugs of abuse through their ability to induce Pavlovian associations with cues of the environment ${ }^{7,8}$. In Pavlovian terms, the drug is an unconditioned stimulus since it can elicit approach or withdrawal if it is rewarding or aversive, respectively. Continuous pairing of the drug with various neutral stimuli, that themselves do not elicit any response, can lead to approach or withdrawal merely upon presentation of the previously neutral, but after pairing, so called conditioned stimuli ${ }^{9}$. CPP analysis is usually performed in an apparatus containing two compartments of the same size but where each compartment is defined by distinct characteristics, such as floor texture, wall patterns and illumination (neutral stimuli). The two compartments are connected either by a corridor or an opening between the compartments. During conditioning, the subject, usually a small rodent, receives passive injections of a drug while restricted to one of the two main compartments and saline while restricted to the other compartment. The rewarding effects of the drug are subsequently assessed in a drug-free session when the subject is allowed to freely explore the whole apparatus. The amount of time spent in the previously drug-paired compartment (the conditioned response) is considered to reflect Pavlovian learning mechanisms mediated between the rewarding effects of the drug and the cues of the compartment associated with its administration (conditioned stimuli). If the animal spends more time in the drug-paired compartment, the drug has induced a conditioned place preference which means that it has rewarding effects on behavior. On the other hand, if the drug is perceived as aversive, the animal will avoid the drug-paired compartment and spend more time in the saline-paired compartment, indicating conditioned place aversion $(\mathrm{CPA})^{8,9,10,11}$

Since optogenetics can be implemented to control neuronal activity in "real-time", the use of a behavioral paradigm similar to, but distinct from, the CPP setup allows for measurement of place preference upon direct neuronal activation. Optogenetics-driven analysis of place preference is therefore often referred to as a real-time place preference (RT-PP) analysis paradigm. In the RT-PP paradigm, optogenetic stimulation of distinct neurons via the Cre-Lox system replaces the systemic delivery of a drug performed in the classical CPP, so that the RT-PP paradigm instead measures if optogenetically induced neuronal stimulation is perceived as rewarding or aversive. The current description will focus on optogenetics mice, but also optogenetics rats can be tested using similar protocols.

Instead of conditioning to one compartment at a time as in the classical CPP paradigm, the optogenetics mouse in the RT-PP paradigm is allowed to move freely in the entire apparatus and behavior is recorded throughout the session. Entry into one of the compartments is paired with intracranial light-stimulation. Under appropriate light stimulation parameters, neurons that express an excitatory opsin will thereby be activated. If the light-stimulation is perceived as rewarding, the optogenetics mouse will remain in the light-paired compartment, while if the light-stimulation is perceived as aversive, the mouse will exit the compartment to escape the stimulation. This type of analysis allows for assessing contingent learning: The subject can trigger light-stimulation and hence neuronal activation by entering a compartment, and stop the stimulation by exiting the compartment, similar to lever-pressing during an instrumental task. Furthermore, associative learning mechanisms can be assessed during subsequent sessions where time spent in each compartment is measured in the absence of stimulation. This way, the researcher can dissociate between the immediately rewarding effects upon stimulation of the neurons of interest and the associative memory formation related to it ${ }^{12}$.

In the current study, we describe two step-by-step setup protocols for optogenetics-driven place preference behavior of freely-moving mice. The first protocol describes RT-PP within a three-compartment apparatus and has been outlined based on the protocols recently presented by Root and colleagues ${ }^{13}$ and other authors ${ }^{12,14,15,16,17,18}$. The experiment consists of two phases comprising several daily sessions (shown in Figure 1A). Each session is designed for different purposes and the parameters of coupling stimulation with a compartment are changed accordingly. The first session, the "Pretest", is used to assess initial preference of the subject to either one of the compartments. While connected to the patch cord, the subject is allowed to freely explore the apparatus in the absence of stimulation for 15 min. If the initial preference to any one compartment is more than $80 \%$, the mouse is excluded from the analysis since initial side bias might skew the analysis. After the "Pretest", "Phase 1" begins. The first part consists of two consecutive, daily, 30 min sessions of "RT-PP". During "Phase 1", the optogenetics mouse is connected to the laser source through the patch cord and placed in the arena to freely explore it. The mouse receives intracranial laser stimulation upon entry into one of the main compartments. Pilot experiments can be performed to determine which compartment will be assigned as laser-paired and which as unpaired. If the stimulation is shown to be rewarding, the laser will be coupled to the least preferred compartment during the "Pretest" and to the most preferred if the stimulation is aversive. Thus, the presented RT-PP protocol follows a biased design in the sense that laser stimulation is not randomly assigned to any of the two main compartments (unbiased design), but is chosen to avoid any initial preference of the mouse. Entry into the other main compartment or the neutral compartment connecting the two main compartments does not give rise to intracranial light stimulation and are thus not light-paired. These sessions allow for real-time assessment of the rewarding or aversive properties of stimulation of specific neuronal populations. On the last day of "Phase 1", a 15 min session without any stimulation takes place. This session serves to address conditioned responses ("CR") which result from associative learning between the stimulation and the environment where it was received. At least three days after "Phase 1", the "Reversal Phase" takes place which follows the same structure as "Phase 1" but the previously non-paired main compartment is now paired with light stimulation. As in the case of "Phase 1", the two stimulation sessions are followed by a "CR" session. The "Reversal Phase" is used to confirm that the behavior of the mouse is contingent upon optogenetic stimulation and not related to random parameters. Each session of the RT-PP experiment has to be separately programmed within the tracking software. The current protocol describes such settings within a specific software, but any other tracking software able to send transistortransistor-logic (TTL) modulation signals to the light source can be used.

The second protocol describes a novel setup termed the Neutral Compartment Preference (NCP) paradigm. This modified protocol of the RTPP takes advantage of the small size and transparency of the connecting corridor which is naturally avoided by the mouse due to its narrow and transparent composition. By pairing both main compartments with light-stimulation and only leaving the corridor free of light-stimulation, the NCP setup can be used to test whether the optogenetic stimulation will force the mouse to spend more time in the corridor to avoid receiving optogenetic stimulation. By comparing the time spent in the two light-paired compartments with the time spent in the corridor, a verification of optogenetically-induced aversion can be made. The NCP experiment consists of two consecutive daily sessions where optogenetics mice 
receive stimulation (30 $\mathrm{min}$ each) to measure preference in real time, and one laser-free session (15 $\mathrm{min}$ ) to assess conditioned responses similarly to the ones in the RT-PP protocol.

The RT-PP and NCP protocols provided below were recently validated in our lab in the study of how different types of neurons located in the ventral tegmental area (VTA) are involved in various aspects of reward-related behavior ${ }^{12}$. Here, to exemplify the implementation of the RT-PP and NCP protocols, dopamine transporter (DAT)-Cre ${ }^{19}$ and vesicular glutamate transporter 2 (VGLUT2)-Cre ${ }^{20}$ transgenic mice were stereotactically injected with AAV carrying a floxed channelrhodopsin2 (ChR2) DNA construct into the VTA whereupon an optic fiber was implanted above the VTA. Behavioral responses obtained upon analysis of these mice using the provided RT-PP and NCP protocols shows how activation of dopaminergic and glutamatergic neurons within the VTA leads to different behavioral responses (Figure 1).

Step-by-step protocols for RT-PP and NCP paradigms are provided with information ranging from genotyping of transgenic mice, stereotaxic viral injections and fiberoptics placement, to programming of tracking software for laser-control and behavioral assessment. In addition, suggestions for modifications of the protocol in terms of stimulation parameters and experimental aspects that can affect the scientific outcome are discussed. While protocols are described in the context of the VTA, they can be applied to any brain area or neuronal population, provided the relevant optogenetics tools, such as relevant Cre-driver and floxed opsins, are available.

\section{Protocol}

This study has been carried out using heterozygous DAT-Cre ${ }^{19}$ and VGLUT2-Cre ${ }^{20}$ mice of both sexes, aged $>8$ weeks and weighing $>20 \mathrm{~g}$. All the experiments were conducted according to the Swedish (Animal Welfare Act SFS 1998:56) and European Union Legislation (Convention ETS 123 and Directive 2010/63/EU) with permission from the local Animal Ethical Committees.

\section{Genotyping of mice}

1. Take ear biopsies using an ear puncher to use for genotyping of the transgenic mice.

2. Prepare the ear punches to perform a polymerase chain reaction $(\mathrm{PCR})$ reaction using purpose-made primers. NOTE: In this protocol, Cre-directed primers were used.

1. Add $75 \mu \mathrm{L}$ of lysis buffer (buffer 1: $250 \mathrm{mM} \mathrm{NaOH}, 2 \mathrm{mM}$ EDTA) in each $1.5 \mathrm{~mL}$ tube containing an ear punch.

2. Incubate in a heating block at $96{ }^{\circ} \mathrm{C}$ for $30 \mathrm{~min}$.

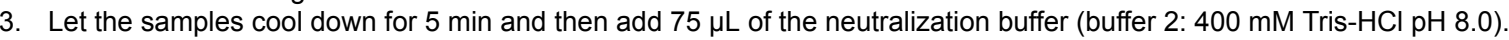

3. Perform PCR according to standard procedures ${ }^{12,21}$ using the appropriate primers (here: Cre FW 5'-ACGAGTGATGAGGTTCGCAAGA-3', Cre REV 5'-ACCGACGATGAAGCATGTTTAG-3').

CAUTION: Work on ice under a PCR hood and pay attention not to contaminate the reagents and the samples.

1. Prepare the PCR master mix. Multiply the following volumes according to how many samples are going to be analyzed, including appropriate control samples. Mix the reagents for a single $25 \mu \mathrm{L}$ final volume reaction in the following order: distilled water (18.9 $\mu \mathrm{L})$, $10 x$ buffer with $\mathrm{MgCl}_{2}(2.5 \mu \mathrm{L}), 10 \mathrm{mM}$ dNTP mix $(0.5 \mu \mathrm{L}), 10 \mu \mathrm{M}$ forward primer $(1 \mu \mathrm{L}), 10 \mu \mathrm{M}$ reverse primer $(1 \mu \mathrm{L}), 5 \mathrm{U} / \mu \mathrm{L}$ DNA polymerase $(0.1 \mu \mathrm{L})$, and template DNA $(1 \mu \mathrm{L}$; will be added in the next steps).

NOTE: Always add a negative, a positive, and an empty (without template DNA) control to ensure valid results.

2. Add $24 \mu \mathrm{L}$ of master mix in PCR tubes.

3. Add $1 \mu \mathrm{L}$ of template DNA (coming from the ear punch from each mouse) in each PCR tube.

4. Centrifuge the PCR tubes briefly to ensure the template DNA is inside the master mix.

5. Perform PCR with a thermal cycler using the cycling program in Table 1.

4. Prepare an agarose gel to run the samples using electrophoresis.

NOTE: The size will depend on the number of samples that need to be analyzed.

1. Add $1 \% \mathrm{w} / \mathrm{v}$ agarose powder in $1 \mathrm{x}$ Tris-acetate-EDTA (TAE) buffer in a glass bottle. Heat in microwave until the agarose is fully dissolved, checking regularly that it does not boil over.

CAUTION: Take precautions to avoid burns.

2. Let the gel cool down to approximately $50^{\circ} \mathrm{C}$ and add a nucleic acid gel stain $(0.5 \mu \mathrm{L} / 50 \mathrm{~mL}$ of gel).

3. Pour the gel in the casting tray containing well combs and leave it in room temperature until it becomes completely solidified. Remove the combs gently.

4. Fill the electrophoresis tank with $1 \times \mathrm{XAE}$ buffer and place the gel in the tank.

5. Add $2 \mu \mathrm{L}$ of $1 \times$ DNA loading dye in each one of the DNA samples.

6. Load $4 \mu \mathrm{L}$ of DNA ladder in the first well of the gel, then proceed to load the full volume of the samples in the remaining wells.

7. Set the power source of the electrophoresis to $140 \mathrm{~V}$ and run for 25-30 min.

8. Place the gel under a UV source and take a picture of the results.

\section{Stereotaxic surgery}

1. After genotyping, separate mice keeping the ones positive for Cre. Wait until they are at least 8 weeks old and weigh $>20 \mathrm{~g}$ to perform surgery.

1. Sanitize the environment and sterilize the surgical tools to perform surgery under aseptic conditions.

2. Inject the mice subcutaneously with analgesic 30 min before surgery.

3. Anesthetize the mice with isoflurane (2-3\% in normal air for induction and $1.5-2.0 \%$ for maintenance of anesthesia). Ensure adequate anesthesia level is achieved by testing the absence of pain reflexes by gently pinching the toe of the mouse. Adjust the isoflurane delivery accordingly. 
4. Place the mouse on the stereotaxic apparatus. Add eye lubricant to prevent eye lesioning due to dryness and shave the hair of the top of the skull. Use a heating pad to maintain the temperature of the mouse stable.

5. Inject $100 \mu \mathrm{L}$ of local anesthetic under the skin of the skull and allow $5 \mathrm{~min}$ to take effect.

6. Prepare the incision site by three circular applications of alcohol or sterile saline alternating with iodine. Use a sterile cotton tip and initiate the application from the incision line, outward.

7. Gently lift the skin with forceps, and make an incision of $\sim 1.5 \mathrm{~cm}$ along the rostrocaudal axis with surgical scissors to reveal the surface of the skull.

8. Using a cotton stick, apply $\mathrm{H}_{2} \mathrm{O}_{2}$ solution to remove the periosteum.

9. Rinse the skull with sterile saline and dry it using sterile cotton tip applicators.

10. Locate the bregma and lambda.

11. Ascertain flat skull alignment by positioning the tip of the injection needle, adjusted on the stereotaxic frame, on bregma and lambda. Measure the ventral coordinates for each position and compare. When the skull is flat the ventral coordinate for both bregma and lambda are identical. If not, adjust the head position and take the measurements again.

12. Find and mark the position (AP: $-3.45 \mathrm{~mm}$, ML: $-0.2 \mathrm{~mm}$ from bregma according to Franklin and Paxinos ${ }^{22}$ ) where the injection of the Cre-dependent virus and the implantation of the optic fiber will take place and make a small hole using a micro drill.

13. Load $400 \mathrm{~nL}$ of virus in the $10 \mu \mathrm{L}$ syringe mounted on the stereotaxic apparatus using a precision pump.

14. Lower the needle (34 G, beveled) carefully and inject $300 \mathrm{~nL}$ of Cre-dependent optogenetic virus (AAV5-EF1a-DIO-ChR2(H134)eYFP $\left[5.6 \times 10^{12} \mathrm{vg} / \mathrm{mL}\right]$ ) in the VTA (AP: $-3.45 \mathrm{~mm}$, ML: $-0.2 \mathrm{~mm}$ from bregma and $-4.4 \mathrm{~mm}$ from the surface of the skull, according to Franklin and Paxinos ${ }^{22}$ ) at $100 \mathrm{~nL} / \mathrm{min}$ injection rate using the precision pump.

15. After injection, leave the needle in place for an additional 10 min to allow for diffusion of the virus (Figure 2A).

16. Retract the needle slowly from the injection site.

17. Make small holes using a microdrill to fit anchor screws that will stabilize the optic fiber and dental cement complex.

18. Take the bregma coordinates again and implant the optic fiber $(200 \mu \mathrm{m}$ diameter, $0.37 \mathrm{NA})$ at: AP: $-3.45 \mathrm{~mm}, \mathrm{ML}:-0.2 \mathrm{~mm}$ from bregma and $-4.0 \mathrm{~mm}$ from the surface of the skull (Figure 2B) according to Franklin and Paxinos ${ }^{22}$.

19. Secure the fiber on the skull using dental cement. Apply enough cement around the optic fiber ferule to secure it to the skull but pay attention to leave 3-4 $\mathrm{mm}$ of the top of the ferule free of cement to allow connection of the patch cord (Figure 2C). NOTE: Pay attention not to fill the hole with cement as this can cause brain tissue damage. Hemostatic materials can be added in the hole to prevent this from happening.

20. Use tissue glue or absorbable sutures to close any open wound and leave the animal to recover for at least two weeks. Give an additional dose of analgesic 12-24 $\mathrm{h}$ after the surgery.

\section{Setting up the control of the laser source}

1. Use single board microcontrollers to control the laser source. Write a script using the appropriate software. Load the script on the microcontroller board using the appropriate connection cable to the computer.

NOTE: The script should include external modulation (input) coming from the tracking software through a TTL box, and an output to the laser to control stimulation parameters. For $10 \mathrm{~ms}$ pulse width at $20 \mathrm{~Hz}$ frequency, use the script found in the Supplemental Coding File.

2. Connect the board to the laser and the TTL box of the tracking hardware.

1. Use a network cable to connect the TTL box to the board (pin 5 for the provided script) (Figure 3A,C).

2. Ensure the laser is set to control by external modulation and connect the laser to the board using an FC/PC cable (pin 13 for the given script) (Figure 3B,C).

3. Connect the appropriate pins to ground parts of the board.

3. Connect the laser source to the optic fiber.

1. Connect the laser source to a rotary joint (Figure 3D).

2. Connect a patch cord (Figure $3 \mathrm{E}$ ) to the rotary joint.

3. Stabilize the rotary joint above the apparatus but outside the recording area. Make sure the length of the fiber-optic patch cord is appropriate to allow the mouse to move without difficulties in the arena (Figure $3 F$ ).

\section{Setting up the experiment for the RT-PP approach within the tracking software}

1. Calibrate the arena setup. Use a ruler to measure a specific part of the physical apparatus, draw a line corresponding to the part measured on the image within the software under the Draw Scale to Calibrate tab and enter the already known value (step 1 in Figure 4).

2. Design the arena. Draw the area where the movement of the mice will be recorded (step 2 in Figure 4).

3. Create the zones. Draw the zones that will eventually be assigned as laser-paired, laser-unpaired and "neutral" (step 3 in Figure 4).

4. Validate the setup to confirm that there are no conflicting parameters, for example zones outside the arena (step 4 in Figure 4)

5. Set the experimental parameters under the tab trial control settings (step 5 in Figure 4).

1. Set the trial time as shown in step 1 in Figure $\mathbf{5}$ for a 30 min RT-PP session.

2. Making sure "hardware control" is enabled, assign a compartment as laser-paired in which entry of the mouse will trigger a TTL signal through the tracking software to the microcontroller board. In Figure 5 (step 2) the laser-paired compartment is compartment A. For the reversal phase, switch the compartments so compartment $B$ will be laser paired and compartment $A$ will be unpaired. Do so by replacing $A$ with $B$ and $B$ with $A$ in the software. 


\section{Modification of the setup to test the aversive properties of the stimulation using the NCP approach}

1. Follow steps 4.1-4.4 as described previously.

2. Set the time of the experiment to 30 min through the "Repeat till" option in the "Reference" box settings (step 1 in Figure 6 ).

3. Assign both A and B zones as laser paired (step 2 in Figure 6) by adding "when center-point is in any of Zone A and Zone B" for the condition box related to the settings for $A$ and $B$ compartments. Note that the laser will turn off when the animal is located in the neutral compartment.

\section{Performing an experiment using laser stimulation}

1. Set up the detection settings.

1. Use a dummy to resemble the mouse in order to ensure appropriate detection settings.

2. Place the dummy in one compartment of the apparatus and use automated setup with dynamic subtraction.

3. Remove the dummy and place it to the opposite compartment. Make sure the dummy is fully detected and if not, adjust the settings via the software to achieve proper detection.

1. During this step also check if the stimulation works as it is supposed to. Start acquisition using the previously configured trial control settings and place the dummy in the laser paired compartment and see if the stimulation is triggered as it should. Then place the dummy in the unpaired and/or the neutral compartment and see if the stimulation is stopped.

2. Use a power meter with a sensor to set the laser power to $10 \mathrm{~mW}$ using the knob on the laser (Figure 3B). Perform this step each time laser stimulation is used.

CAUTION: Use protective eye equipment as direct exposure to laser light can cause permanent eye damage.

3. Place the mouse in the apparatus.

1. Gently take the mouse out of its cage and connect the fiber-optic implant to the fiber-optic patch cord using a ceramic sleeve.

2. Place the mouse gently in the neutral compartment of the three-compartment apparatus.

3. Wait until the mouse is detected by the software.

4. Remove the vertical sliding doors restricting the animal from entering the main compartments.

5. Allow the animal to explore freely without any disturbances.

NOTE: The same procedure is followed when the animal is not receiving stimulation with the exception that step 6.2 is not needed and that the laser is off the whole time.

\section{Representative Results}

The three-compartment apparatus (Figure $\mathbf{3 F}$ ) is suitable to address the rewarding effects of drugs and to assess in real time the rewarding or aversive properties of direct stimulation of neurons using optogenetics. It consists of two main compartments $(20 \mathrm{~cm}[\mathrm{~W}] \times 18 \mathrm{~cm}[\mathrm{~L}] \times 25 \mathrm{~cm}$ $[\mathrm{H}])$ and one smaller connecting compartment $(20 \mathrm{~cm}[\mathrm{~W}] \times 7 \mathrm{~cm}[\mathrm{~L}] \times 25 \mathrm{~cm}[\mathrm{H}])$. The main compartments have distinct wall and floor texture and patterns in order to facilitate associative learning, while the connecting/neutral compartment is narrow and transparent so the mice spend naturally less time in it. As described above, the tracking software can be used to record several behavioral parameters of the mice including movement and time spent in each compartment, and to control the laser stimulation. The entire RT-PP experiment takes place throughout 8 sessions (Figure 1A) and allows both the assessment of the rewarding or aversive properties of the direct stimulation (days $3,4,6$, and 7 ) and the formation of associations, positive or negative, in response to previous experience (days 5 and 8 , "CR").

Firstly, we tested DAT-Cre mice injected with AAV-ChR2-eYFP virus in the VTA to target dopaminergic neurons. In accordance with the literature, we observed that the mice preferred to spend time in the compartment paired with the stimulation (Figure 1B, phase 1, days 3 and 4, blue circles, two-way repeated measures [RM] analysis of variance [ANOVA], effect of compartment $F_{(2,18)}=141, p<0.001$; effect of session $x$ compartment $\mathrm{F}_{(12,108)}=42.1, p<0.001$; Tukey's post hoc test paired vs unpaired $\left.p<0.001\right)$. The reversal phase, where the pairing of the compartments to laser stimulation was switched, confirmed these observations (Figure 1B, days 6 and 7, blue circles, Tukey's post hoc test paired vs unpaired compartment $p<0.001$ ) thus excluding the possibility that the results obtained from phase 1 were related to side biases or random parameters. Averaging the time spent in each compartment during the four days of RT-PP confirmed that the mice spent on average about $70 \%$ of their time in the laser paired compartment as opposed to the unpaired $(\sim 20 \%)$ and the neutral ( 10\%) compartments (Figure 1B bar graph, one-way RM ANOVA, effect of stimulation $\mathrm{F}_{(2,6)}=139, p<0.001$, Tukey's post how paired vs unpaired and neutral compartments $p<$ 0.001 ). Furthermore, in the absence of stimulation, on days 5 and 8 , the mice spent significantly more time in the compartment previously paired with laser stimulation (Tukey's post hoc $p<0.001$ ), indicating that the previous experience was sufficient to induce associative learning behaviors reflected as "seeking" of the stimulation. These data are in accordance with the literature and demonstrate that the current method can be used reliably to investigate the rewarding effects of optogenetic stimulation of specific neuronal populations in the VTA. 
We then tested VGLUT2-Cre mice injected with AAV-ChR2-eYFP in the VTA as above, to target glutamatergic neurons of the VTA. In this experiment, we observed an opposite behavioral phenotype from the one demonstrated by the DAT-Cre mice. Thus, the mice avoided the compartment paired to the stimulation and spent more time in the unpaired during all RT-PP days (Figure 1C left, two-way RM ANOVA, effect of compartment $F_{(2,12)}=40.9, p<0.001$; effect of session $x$ compartment $F_{(12,72)}=16.1, p<0.001$; Tukey's post hoc test paired vs unpaired $p<0.001$; Figure 1C right, one-way RM ANOVA effect of stimulation $\mathrm{F}_{(2,6)}=162, p<0.001$, Tukey's post hoc paired vs unpaired and neutral compartments $p<0.001$ ). Interestingly, during the "CR" days 5 and 8 , the mice did not show a clear avoidance of the previously paired compartment (no differences between paired and unpaired compartments). It is possible that the lack of conditioned response is due to inadequate time spent in the laser paired compartment, which prevented the formation of associations between laser activation and the particular environment where that took place. To further explore this avoidance phenotype, we used a modified protocol which we named "neutral compartment preference", abbreviated NCP. In this experiment, both main compartments were paired to stimulation and the neutral compartment remained stimulation-free (Figure 7A). We hypothesized that, if the stimulation has aversive properties, then the mouse will be forced to spend time in the smaller, neutral compartment, to avoid it. Indeed, in both days of stimulation (Stim1 and Stim2) the mice spent the majority of the time in the neutral compartment (about 80\%) compared to the paired compartments (Figure 7B,C; left: two-way RM ANOVA effect of compartment $F_{(2,8)}=70.9, p<0.001$; effect of session $x$ compartment $F_{(4,16)}=6.9, p=0.002$, Tukey's post hoc "Stimulation 1" neutral compartment vs compartment 1 and $2 p<0.01$, "Stimulation 2" neutral compartment vs compartment 1 and $2 p<0.001$; right: one-way RM ANOVA, effect of stimulation $\mathrm{F}_{(2,2)}=54.2, p=0.018$, Tukey's post hoc test paired 1 and 2 vs neutral $\left.p<0.05\right)$. As in the case of "CR" days during the RT-PP test, the mice did not seem to form negative associations between the compartments and the stimulation; that is, in the absence of stimulation (CR), they explored all the compartments to the same degree (Figure 7B, no differences between time spent in paired compartments and neutral compartment). The results of these experiments confirmed the behavioral phenotype observed during the RT-PP setup and thereby support the combinatorial implementation of both the RT-PP and NCP paradigms.

A

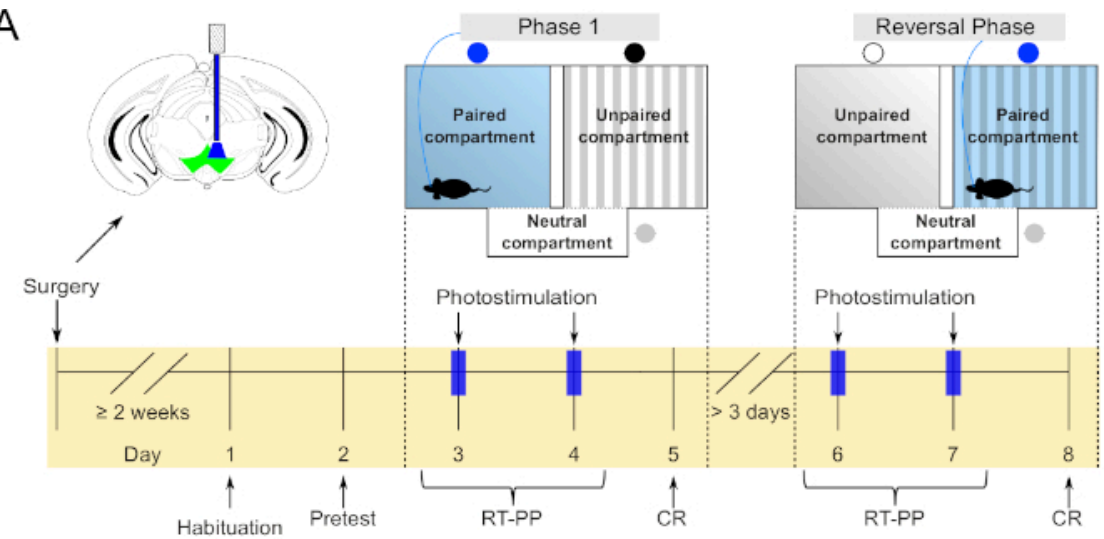

B
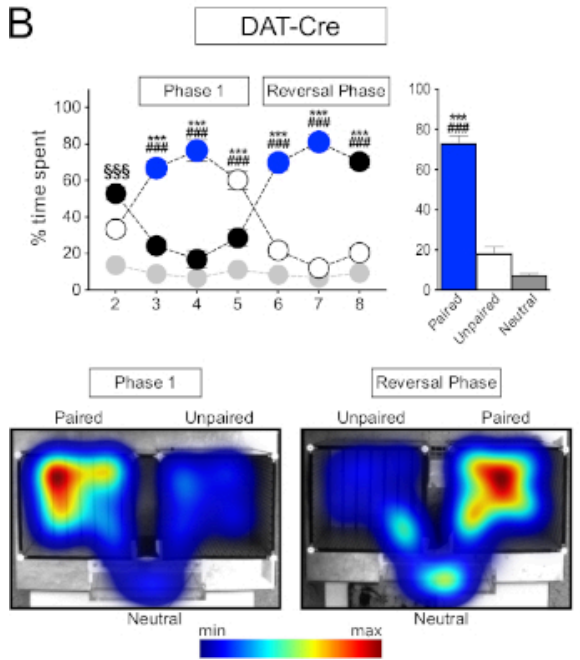

C

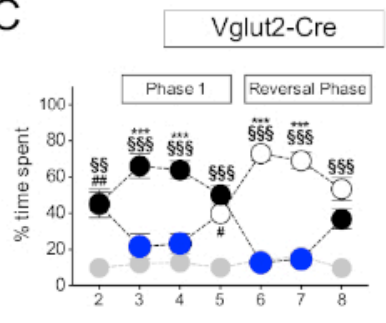

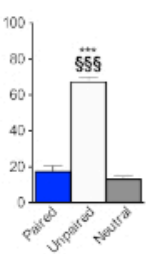

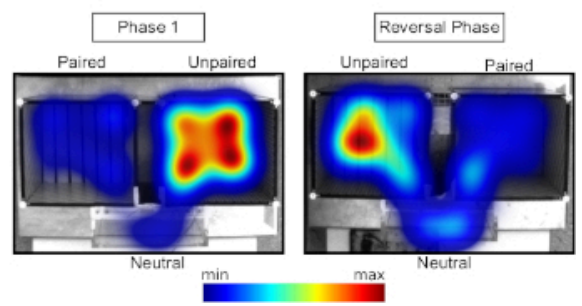

Figure 1: Behavioral testing using optogenetics in the RT-PP paradigm. (A) Schematic representation of the timeline of the experiments. $(B, C)$ Top left: graph representing the percentage of time spent in each compartment throughout the RT-PP experiment for DAT-Cre $(\mathrm{N}=10)$ and VGLUT2-Cre ( $\mathrm{N}=7)$ mice injected with AAV-ChR2-eYFP. Blue circles: laser-paired compartment; white, black circles: main compartments; gray circles: neutral compartment. Top right: average percentage of time spent in each compartment during days 3, 4, 6, and 7 (RT-PP). Bottom: representative heatmaps of time spent in each compartment for a DAT-Cre and a VGLUT2-Cre mouse. All data were normally distributed

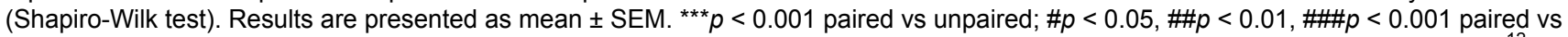
neutral compartment; $\S \S p<0.01, \S \S \S p<0.001$ unpaired vs neutral compartment. This figure has been modified from Bimpisidis et al. ${ }^{12}$. Please click here to view a larger version of this figure. 

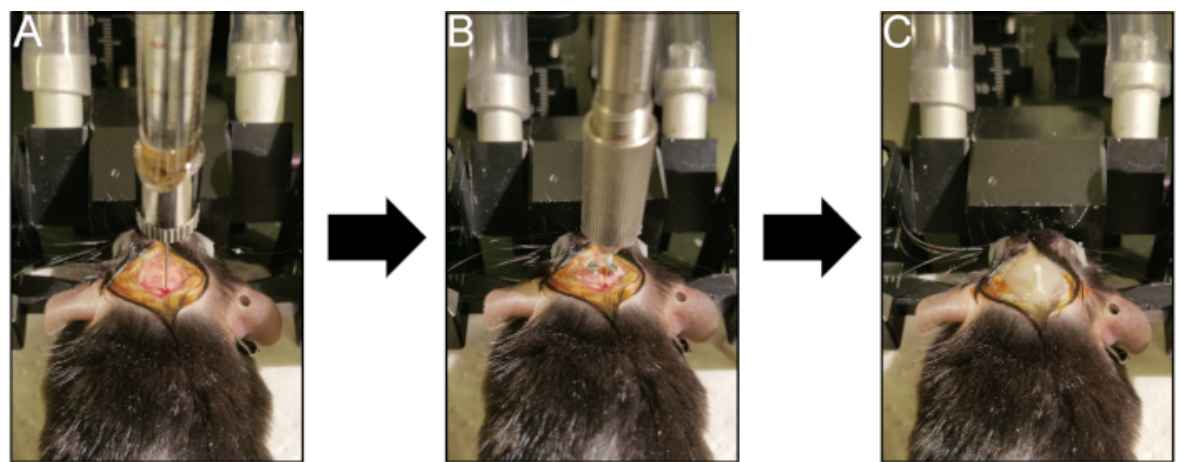

Figure 2: Surgical procedure for optogenetic experiments. (A) Injection of Cre-dependent viral vector in the VTA. (B) Implantation of the optic fiber above the injection site. Note the anchor screws used for stabilization. (C) Permanent anchoring of the fiber on the skull using dental cement. Please click here to view a larger version of this figure.

A

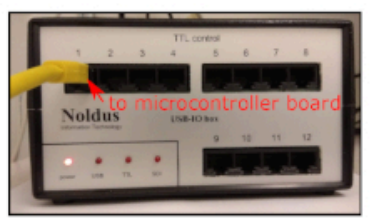

B

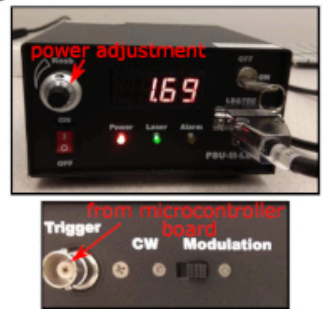

E

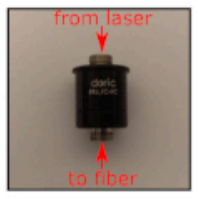

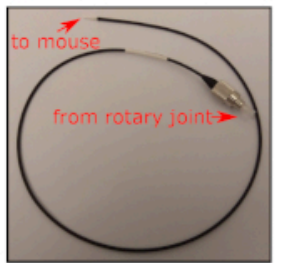

C

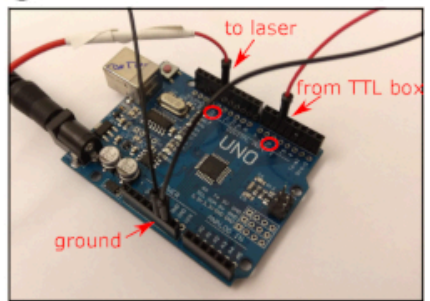

F

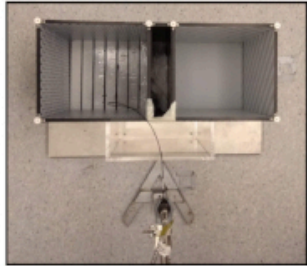

Figure 3: Equipment used in the optogenetics experiments. (A) The TTL box used in the studies. It receives input from the tracking software and sends TTL signals to the microcontroller board. (B) Front (top) and rear view (bottom) of the laser source used for the experiments. (C) The microcontroller board used to control the laser stimulation. Note the connections from the TTL box and to the laser source. (D) Rotary joint. (E) Fiber-optic patch chord used in the experiments. (F) The three-compartment apparatus used for RT-PP and NCP experiments. Please click here to view a larger version of this figure.

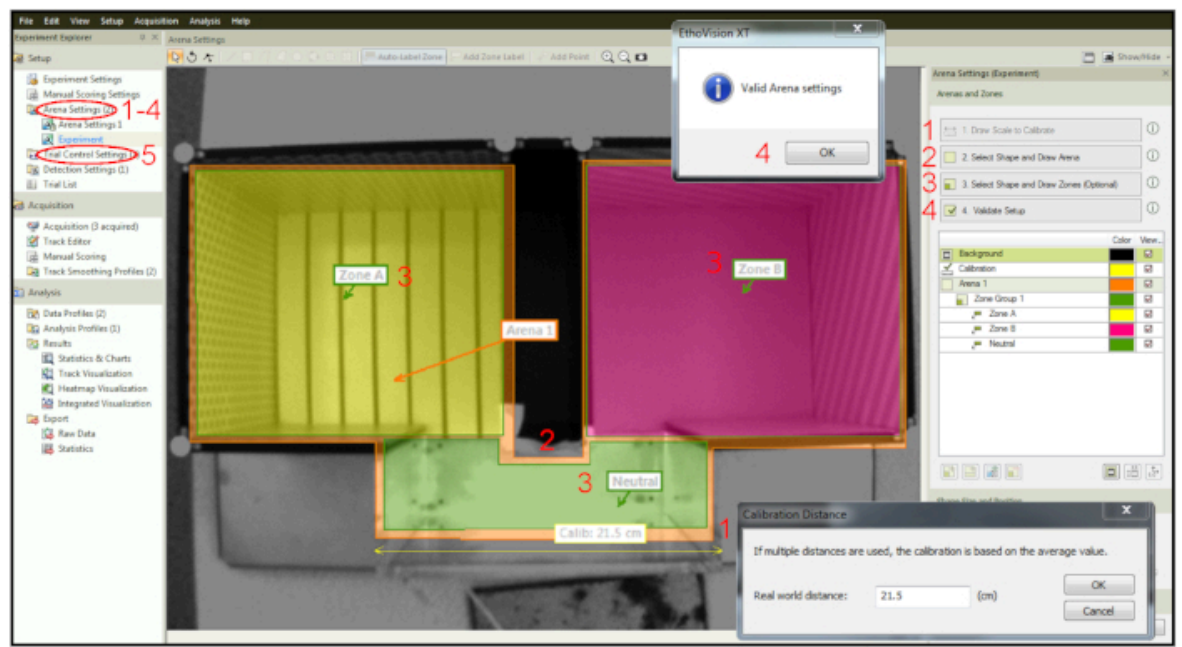

Figure 4: Designing arena and zones within the tracking software. Step 1: Calibration of the setup. Step 2: Drawing of the whole arena. Step 3: Drawing zones within the arena. Step 4: Setup validation. Step 5: Trial Control Settings tab for setting up time and stimulation parameters. Please click here to view a larger version of this figure. 


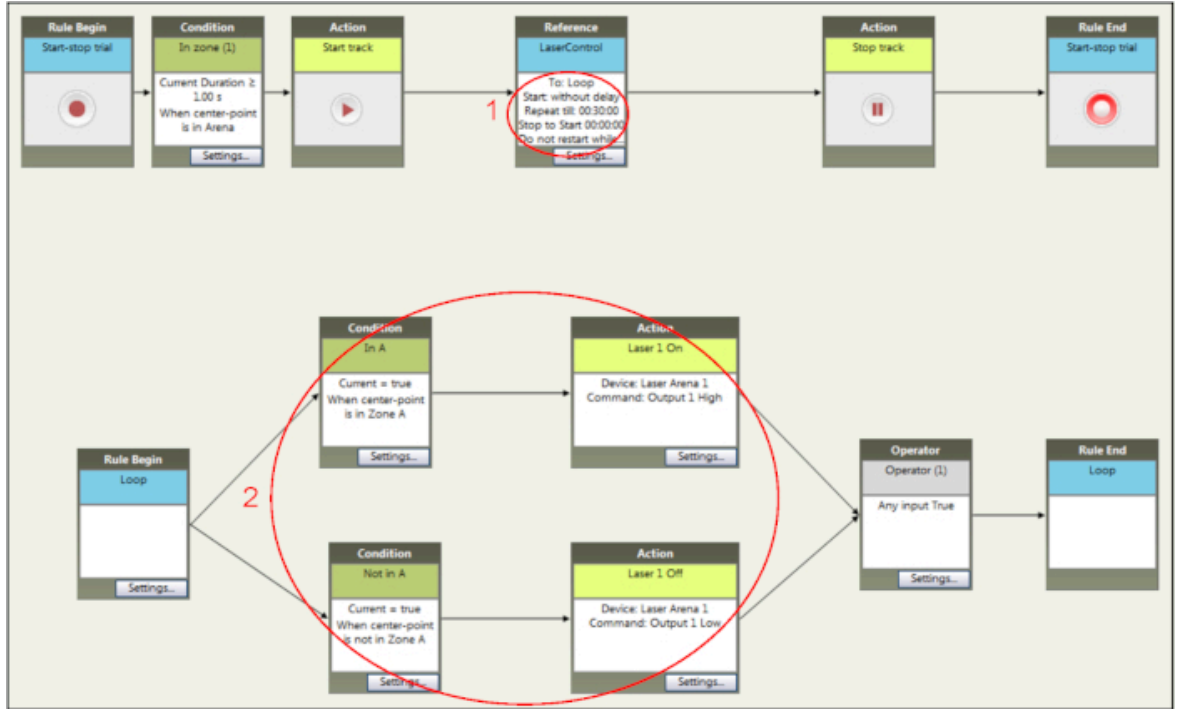

Figure 5: Setting up time and stimulation parameters of an RT-PP experiment within the tracking software. Adding specific rules for the duration (step 1) and the conditions for light stimulation (step 2). The conditions can be easily changed to fit the requirements for the reversal phase. Please click here to view a larger version of this figure.

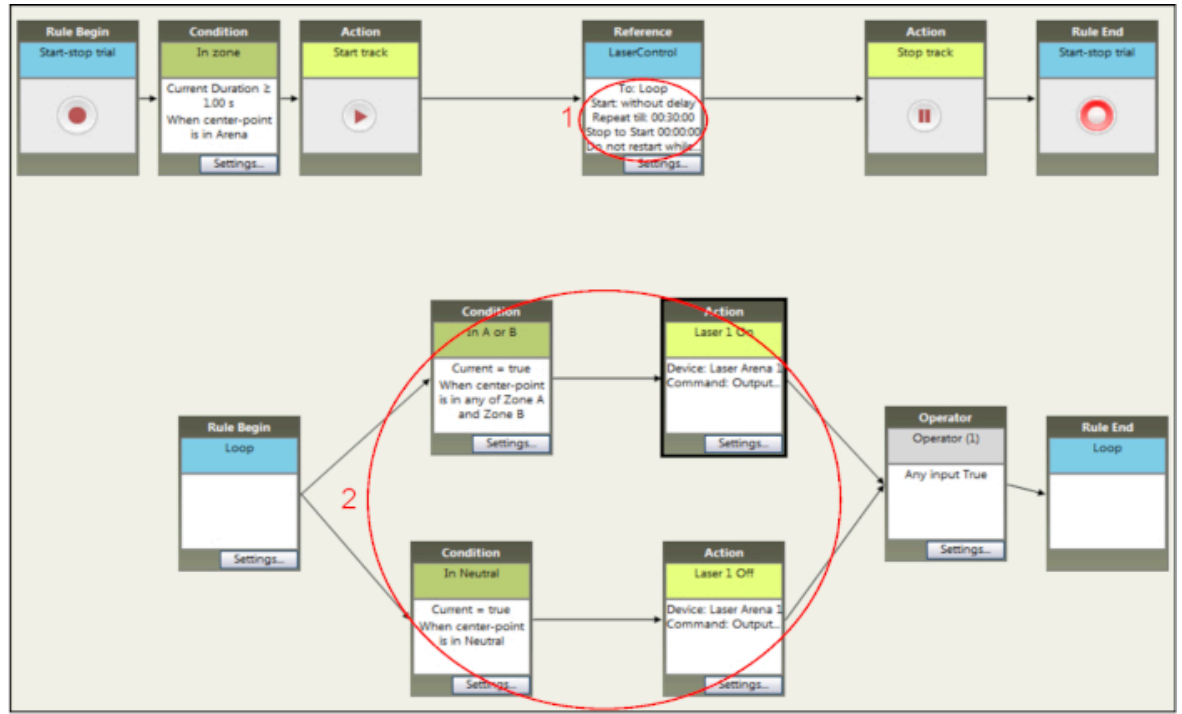

Figure 6: Setting up time and stimulation parameters of NCP experiments within the tracking software. The duration of the stimulation sessions (step 1) is similar to the ones for RT-PP but the conditions for light stimulation activation (step 2) are different. Entry to either main compartment (here named zone A and zone $B$ ) results in optogenetic stimulation that is terminated only when the mouse enters the neutral compartment. Please click here to view a larger version of this figure. 

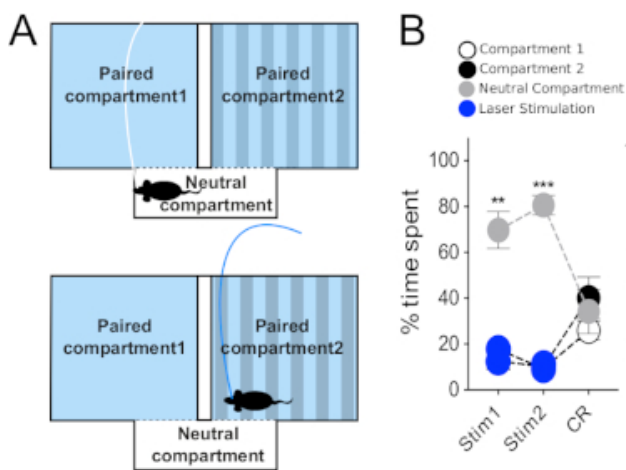

C
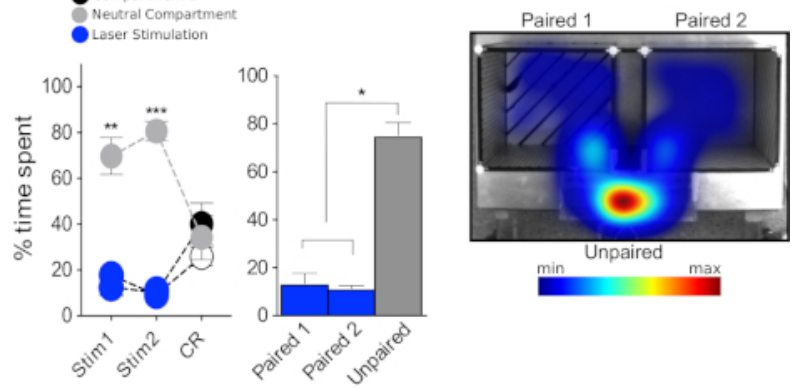

Figure 7: Behavioral testing using optogenetics in the NCP paradigm. (A) Schematic representation of the experimental setup. (B) Left: graph representing the percentage of time spent in each compartment during the two days of stimulation (Stim1 and Stim 2) and during the conditioned response (CR) session for VGLUT2-Cre mice injected with AAV-ChR2 in the VTA (N = 5). Right: average percentage of time spent in each compartment during the two days of stimulation of the NCP. (C) Representative heatmap of time spent in each compartment for a VGLUT2Cre mouse during one of the stimulation days. Data were normally distributed (Shapiro-Wilk test). Results are presented as mean \pm SEM. ${ }^{*} p<$ $0.05,{ }^{* *} p<0.01,{ }^{* \star *} p<0.001$ unpaired vs paired 1 and paired 2 compartments. This figure has been modified from Bimpisidis et al. ${ }^{12}$. Please click here to view a larger version of this figure.

\begin{tabular}{|l|l|l|l|}
\hline Step & Temperature & Duration & Cycles \\
\hline 1. Initial denaturation & $95^{\circ} \mathrm{C}$ & $4 \mathrm{~min}$ & 1 \\
\hline 2. Denaturation & $95^{\circ} \mathrm{C}$ & $30 \mathrm{~s}$ & 30 \\
\hline 3. Annealing & $55^{\circ} \mathrm{C}$ & $30 \mathrm{~s}$ & \\
\hline 4. Extension & $72^{\circ} \mathrm{C}$ & $40 \mathrm{~s}$ & 1 \\
\hline 5. Final extension & $72^{\circ} \mathrm{C}$ & $6 \mathrm{~min}$ & 1 \\
\hline 6. Hold & $4^{\circ} \mathrm{C}$ & Until stopped by the experimenter & 1 \\
\hline
\end{tabular}

Table 1: The PCR cycling program.

Supplemental Coding File. Please click here to view this file (Right click to download).

\section{Discussion}

In the current study, we present two step-by step protocols of how to perform different types of place preference analyses using optogenetics in mice. The protocols outlined were used to assess the rewarding or aversive behavioral phenotypes of VTA neurons (Figure 1 and Figure 6 ) ${ }^{12}$, but can be utilized to explore the behavioral role of neurons in other brain regions as well.

Several recent studies have described RT-PP paradigms in two-compartment ${ }^{23,24}$ and three-compartment apparatuses ${ }^{13,14,15,16,17,18}$. The current protocols describe detailed setups for the RT-PP and NCP protocols in a three-compartment apparatus resembling those traditionally used in CPP experiments to assess behavioral effects upon administration of drugs of abuse. While the results are only presented here as the percentage of time the mouse spent in each compartment, the tracking software does allow for analysis of several other behavioral parameters, such as transitions to zones, velocity, time spent immobile and more. Analysis of different parameters can be of importance to the interpretation of data.

The current RT-PP protocols are flexible and can be modified to test if different types of stimulation patterns have rewarding effects. The parameters of laser control can be easily changed either through the script of the microcontroller board or within the tracking software, demonstrating the versatility of the setup. We suggest a $20 \mathrm{~Hz}$ stimulation frequency which is within the range, and sometimes lower, of frequencies applied in previous studies using the same opsin variant (ChR2/H134R) to study dopaminergic and glutamatergic neurons and their terminals $^{13,14,16,17,18,23,24,25,26,27}$. Recent studies have demonstrated that higher stimulation frequencies can have the opposite effects on behavior than lower ones, and that these effects are mediated through a depolarization block caused by higher frequencies ${ }^{28}$. Similarly, differences in behavioral output have been shown when stimulating glutamatergic and GABAergic neurons in the lateral preoptic area ${ }^{15}$. These studies examined neurons of different areas than the VTA and the largest effects were observed on high frequencies of non-glutamatergic neurons ${ }^{15,28}$ Our choice on $20 \mathrm{~Hz}$ is based on previous studies of glutamatergic and dopaminergic VTA neurons demonstrating that by varying stimulation frequencies, reward-related behavioral output is not significantly altered ${ }^{24,26}$.

An additional parameter that can be adjusted and which may influence the experimental outcome is the power of the light source. Higher laser power can increase the size of the light-stimulated area, which may be beneficial in some types of experiments but with the drawback of an increase in temperature ${ }^{5}$. Indeed, a recent study has demonstrated that laser-induced increases in temperature can alter brain physiology and affect behavioral measurements ${ }^{29}$. These observations highlight the importance of including opsin-negative controls in the experimental design In the current protocol, we used $10 \mathrm{~mW}$ laser power that is similar and has been previously shown to be effective in stimulating dopaminergic and glutamatergic neurons in the VTA ${ }^{16,24,26}$. When setting up experiments, it is important to pay attention to the size of the area in which the cells of interest are located and the fiber-optics and patch cord properties (numerical aperture, core diameter). These parameters are essential to 
take into consideration when performing calculations related to laser power. For details, the calculator developed by Karl Deisseroth's lab (http:// web.stanford.edu/group/dlab/cgi-bin/graph/chart.php) can be used.

Histological verification of the Cre-Lox recombination is another critical aspect when applying optogenetics experiments. Validation of the recombination efficiency should always take place in a pilot cohort before the initiation of any behavioral experiments in a large group of animals. This is important for ethical reasons but also for optimized experimental output. Each viral construct might show variable specificity for distinct neuronal types and in different regions ${ }^{5}$, a parameter which can affect the experiments in unpredictable and even misleading ways. For instance, we have previously validated the Cre-Lox recombination pattern of $A A V 5$ viruses in the VTA of DAT-Cre mice and found that unilateral injections were sufficient to target the majority of the area of interest. When we then studied spatially restricted subpopulations within the VTA, such as one characterized by NeuroD6 expression, we observed that bilateral viral injections were more efficient to target larger number of neurons giving more pronounced behavioral effects upon optogenetic light-stimulation ${ }^{12}$. Furthermore, the time from surgery to initiation of behavioral experiments has to be addressed carefully. Two weeks is enough time for a ChR2 DNA construct to be expressed in cell bodies as we show here, but longer waiting times ( $\sim 8$ weeks) might be needed if the investigator is testing the effect of stimulation in projection areas ${ }^{13,14,15,17}$.

It worth noting that the volume of virus injected (in our case $300 \mathrm{~nL}$ ) might be suitable when studying neurons in the VTA, but volume and titer must be adjusted depending on efficiency of transduction and the size of the structure studied. Additionally, for bilateral structures located at a distance from the mediolateral axis, it might be necessary to perform bilateral injections, and to also implant fiberoptics bilaterally to ensure activation/inhibition in both hemispheres.

Finally, it is always necessary to perform post-mortem histological analysis to validate and confirm the efficiency of the Cre-Lox recombination and to verify the correct implantation site of the optic fiber at the intended location. Unexpected, over-restricted or excessive Cre-Lox recombination might occur due to unknown distribution of neurons expressing Cre outside the borders of the intended area, or due to differences in the virus serotype, poor handling of the virus, clogging of the syringe for virus delivery or other surgery-related problems. Verification of satisfactory Cre-Lox recombination and correct fiberoptics-implantation has to be performed to confirm any statistical results of the behavioral assessments in order to draw safe conclusions.

In terms of the data provided here as examples of how the two behavioral paradigms can be used, the significant preference to the light-paired side obtained by optogenetic stimulation of dopaminergic neurons in the VTA by analyzing DAT-Cre mice in the RT-PP paradigm was expected based on previous findings ${ }^{23,24,25,26,27}$ while the avoidance of this side shown by the VGLUT2-Cre mice was not anticipated. VGLUT2 neurons of the VTA and their projections have been shown to be involved in both reward and aversion ${ }^{16,17,24,30,31}$, and we therefore performed the NCP analysis to assess the apparent avoidance behavior observed in the current RT-PP setup in more detail. By using the narrow, transparent corridor as the only non-light paired compartment to confirm the aversive properties of stimulation of VTA glutamatergic neurons, it is evident that in this particular three-compartment setup, optogenetic activation of these neurons causes an aversive response. These experiments, which were shown here to exemplify situations that might benefit from using both the RT-PP and NCP protocols, were part of a recently published study, and the complete data set as well as discussions regarding these findings can be found in this publication ${ }^{12}$.

In addition to the NCP, alternative ways to confirm aversion include the strong illumination of an area within an open field area while pairing the rest of the arena to laser activation, or perform an active avoidance task in which the mouse has to perform a specific pattern of behavior to terminate the laser stimulation ${ }^{15}$.

To summarize, the protocols described provide critical information of how to successfully perform RT-PP and NCP analysis in the most efficient way in order to unravel the role of neuronal activation in reward and aversion. Depending on the scientific hypothesis, a range of parameters can be analyzed using these protocols, and each protocol can also be combined with other validated paradigms for optimized behavioral analyses implementing optogenetics to address specific brain areas and neurons of interest.

\section{Disclosures}

The authors have nothing to disclose.

\section{Acknowledgments}

Our funding sources are gratefully acknowledged: Uppsala University, Vetenskapsrådet (Swedish Research Council), Hjärnfonden, Parkinsonfonden, the Research Foundations of Bertil Hållsten, OE\&Edla Johansson, Zoologisk Forskning and Åhlén. Animals were kept at Uppsala University and experiments were performed at the Uppsala University Behavioral Facility.

\section{References}

1. Fenno, L., Yizhar, O., Deisseroth, K. The Development and Application of Optogenetics. Annual Review of Neuroscience. 34 (1), $389-412$ (2011).

2. Kim, C.K., Adhikari, A., Deisseroth, K. Integration of optogenetics with complementary methodologies in systems neuroscience. Nature Reviews Neuroscience. 18 (4), 222-235 (2017).

3. Deisseroth, K. Optogenetics: 10 years of microbial opsins in neuroscience. Nature Neuroscience. 18 (9), $1213-1225$ (2015).

4. Nagy, A. Cre recombinase: The universal reagent for genome tailoring. Genesis. 26 (2), 99-109 (2000).

5. Yizhar, O., Fenno, L.E., Davidson, T.J., Mogri, M., Deisseroth, K. Optogenetics in Neural Systems. Neuron. 71 (1), 9-34 (2011).

6. Pupe, S., Wallén-Mackenzie, A. Cre-driven optogenetics in the heterogeneous genetic panorama of the VTA. Trends in Neurosciences. 38 (6), 375-386 (2015).

7. Spanagel, R. Animal models of addiction. Dialogues in Clinical Neuroscience. 19 (3), 247-258 (2017). 
8. Tzschentke, T.M. Measuring reward with the conditioned place preference (CPP) paradigm: Update of the last decade. Addiction Biology. 12 (3-4), 227-462 (2007).

9. Hoffman, D.C. The use of place conditioning in studying the neuropharmacology of drug reinforcement. Brain Research Bulletin. 23 (4-5), 373-387 (1989).

10. Huston, J.P., Silva, M.A.D.S., Topic, B., Müller, C.P. What's conditioned in conditioned place preference? Trends in Pharmacological Sciences. 34 (3), 162-166 (2013).

11. Bardo, M.T., Bevins, R.A. Conditioned place preference: What does it add to our preclinical understanding of drug reward? Psychopharmacology. 153 (1), 31-43 (2000).

12. Bimpisidis, Z. et al. The NeuroD6 subtype of VTA neurons contributes to psychostimulant sensitization and behavioral reinforcement. eNeuro. 6 (3), e0066-19.2019 (2019).

13. Root, D.H., Mejias-Aponte, C.A., Qi, J., Morales, M. Role of Glutamatergic Projections from Ventral Tegmental Area to Lateral Habenula in Aversive Conditioning. Journal of Neuroscience. 34 (42), 13906-13910 (2014).

14. Steidl, S., Wang, H., Ordonez, M., Zhang, S., Morales, M. Optogenetic excitation in the ventral tegmental area of glutamatergic or cholinergic inputs from the laterodorsal tegmental area drives reward. European Journal of Neuroscience. 45 (4), 559-571 (2017).

15. Barker, D.J. et al. Lateral Preoptic Control of the Lateral Habenula through Convergent Glutamate and GABA Transmission. Cell Reports. 21 (7), 1757-1769 (2017).

16. Wang, H.-L., Qi, J., Zhang, S., Wang, H., Morales, M. Rewarding Effects of Optical Stimulation of Ventral Tegmental Area Glutamatergic Neurons. The Journal of Neuroscience. 35 (48), 15948-54 (2015).

17. Qi, J. et al. VTA glutamatergic inputs to nucleus accumbens drive aversion by acting on GABAergic interneurons. Nature Neuroscience. 19, 725-733 (2016).

18. Qi, J. et al. A glutamatergic reward input from the dorsal raphe to ventral tegmental area dopamine neurons. Nature Communications. $\mathbf{5}$, 5390, (2014).

19. Ekstrand, M.I. et al. Progressive parkinsonism in mice with respiratory-chain-deficient dopamine neurons. Proceedings of the National Academy of Sciences of the United States of America. 104 (4), 1325-1330 (2007).

20. Borgius, L., Restrepo, C.E., Leao, R.N., Saleh, N., Kiehn, O. A transgenic mouse line for molecular genetic analysis of excitatory glutamatergic neurons. Molecular and Cellular Neuroscience. 45 (3), 245-257 (2010).

21. Papathanou, M. et al. Targeting VGLUT2 in Mature Dopamine Neurons Decreases Mesoaccumbal Glutamatergic Transmission and Identifies a Role for Glutamate Co-release in Synaptic Plasticity by Increasing Baseline AMPA/NMDA Ratio. Frontiers in Neural Circuits. 12, 64 (2018).

22. Franklin, K.B.J., Paxinos, G. The mouse brain in stereotaxic coordinates. Academic Press. Boston, MA (2008).

23. Tsai, H.-C. et al. Phasic firing in dopaminergic neurons is sufficient for behavioral conditioning. Science. 324 (5930), 1080-4 (2009).

24. Yoo, J.H. et al. Ventral tegmental area glutamate neurons co-release GABA and promote positive reinforcement. Nature Communications. 7 , 1-13 (2016)

25. Pascoli, V., Terrier, J., Hiver, A., Lüscher, C. Sufficiency of Mesolimbic Dopamine Neuron Stimulation for the Progression to Addiction. Neuron. 88 (5), 1054-1066 (2015).

26. Ilango, A., Kesner, A.J., Broker, C.J., Wang, D. V., Ikemoto, S. Phasic excitation of ventral tegmental dopamine neurons potentiates the initiation of conditioned approach behavior: parametric and reinforcement-schedule analyses. Frontiers in Behavioral Neuroscience. 8, 155 (2014).

27. Kim, K.M. et al. Optogenetic mimicry of the transient activation of dopamine neurons by natural reward is sufficient for operant reinforcement. PLOS ONE. 7 (4), 1-8 (2012).

28. Kroeger, D. et al. Galanin neurons in the ventrolateral preoptic area promote sleep and heat loss in mice. Nature Communications. 9, 4129 (2018).

29. Owen, S.F., Liu, M.H., Kreitzer, A.C. Thermal constraints on in vivo optogenetic manipulations. Nature Neuroscience. 22 (7), $1061-1065$ (2019).

30. Root, D.H., Estrin, D.J., Morales, M. Aversion or Salience Signaling by Ventral Tegmental Area Glutamate Neurons. iScience. 2, 51-62 (2018).

31. Lammel, S. et al. Diversity of transgenic mouse models for selective targeting of midbrain dopamine neurons. Neuron. 85 (2), $429-438$ (2015). 